\title{
Chronic multisite brain recordings from a totally implantable bidirectional neural interface: experience in 5 patients with Parkinson's disease
}

\author{
Nicole C. Swann, PhD, ${ }^{1}$ Coralie de Hemptinne, PhD, ${ }^{1}$ Svjetlana Miocinovic, MD, PhD, ${ }^{2}$ \\ Salman Qasim, BS, ${ }^{1}$ Jill L. Ostrem, MD, ${ }^{2}$ Nicholas B. Galifianakis, MD, ${ }^{2}$ Marta San Luciano, MD, ${ }^{2}$ \\ Sarah S. Wang, PhD, ${ }^{2}$ Nathan Ziman, BS, ${ }^{2}$ Robin Taylor, MSN, FNP, ${ }^{2}$ and Philip A. Starr, MD, PhD ${ }^{1,3,4}$ \\ Departments of ${ }^{1}$ Neurological Surgery and ${ }^{2}$ Neurology; ${ }^{3}$ Kavli Institute for Fundamental Neuroscience; and ${ }^{4}$ Graduate Program in \\ Neuroscience, University of California, San Francisco, California
}

OBJECTIVE Dysfunction of distributed neural networks underlies many brain disorders. The development of neuromodulation therapies depends on a better understanding of these networks. Invasive human brain recordings have a favorable temporal and spatial resolution for the analysis of network phenomena but have generally been limited to acute intraoperative recording or short-term recording through temporarily externalized leads. Here, the authors describe their initial experience with an investigational, first-generation, totally implantable, bidirectional neural interface that allows both continuous therapeutic stimulation and recording of field potentials at multiple sites in a neural network.

METHODS Under a physician-sponsored US Food and Drug Administration investigational device exemption, 5 patients with Parkinson's disease were implanted with the Activa PC+S system (Medtronic Inc.). The device was attached to a quadripolar lead placed in the subdural space over motor cortex, for electrocorticography potential recordings, and to a quadripolar lead in the subthalamic nucleus (STN), for both therapeutic stimulation and recording of local field potentials. Recordings from the brain of each patient were performed at multiple time points over a 1-year period.

RESULTS There were no serious surgical complications or interruptions in deep brain stimulation therapy. Signals in both the cortex and the STN were relatively stable over time, despite a gradual increase in electrode impedance. Canonical movement-related changes in specific frequency bands in the motor cortex were identified in most but not all recordings.

CONCLUSIONS The acquisition of chronic multisite field potentials in humans is feasible. The device performance characteristics described here may inform the design of the next generation of totally implantable neural interfaces. This research tool provides a platform for translating discoveries in brain network dynamics to improved neurostimulation paradigms.

Clinical trial registration no.: NCT01934296 (clinicaltrials.gov)

https://thejns.org/doi/abs/10.3171/2016.11.JNS161162

KEY WORDS deep brain stimulation; DBS; Parkinson's disease; PD; brain-machine interface; basal ganglia; motor cortex; electrophysiology; functional neurosurgery

$\mathrm{M}$ UCH about brain networks in Parkinson's disease (PD) has been learned from invasive brain recordings in patients undergoing neurosurgery in the awake state. Intraoperatively studied signals include basal ganglia single-unit discharge, ${ }^{44}$ basal ganglia local field potentials (LFPs) ${ }^{24}$ and motor cortex electrocorticog- raphy $(\mathrm{ECoG}){ }^{25}$ LFPs and ECoG potentials mainly reflect summed, synchronized synaptic activity from neuronal populations close to the recording electrode. Local field potentials have also been studied through temporarily externalized basal ganglia deep brain stimulation (DBS) leads, allowing recording for several days postoperatively. ${ }^{15}$ To-

ABBREVIATIONS DBS = deep brain stimulation; ECoG = electrocorticography; EKG = electrocardiogram; FDA = Food and Drug Administration; IPG = implanted pulse generator; LFP = local field potential; PD = Parkinson's disease; PSD = power spectral density; RMS = root mean square; STN = subthalamic nucleus; UPDRS = Unified Parkinson's Disease Rating Scale.

SUBMITTED May 7, 2016. ACCEPTED November 7, 2016.

INCLUDE WHEN CITING Published online April 14, 2017; DOI: 10.3171/2016.11.JNS161162. 
gether, these recording approaches have redefined PD as a circuit disorder characterized by excessive neuronal synchronization, ${ }^{24}$ have led to a mechanistic understanding of the effects of acute DBS, ${ }^{13}$ and have suggested strategies to improve DBS therapy by incorporating feedback control. ${ }^{16}$

However, intraoperative and short-term perioperative recordings from externalized leads suffer from several disadvantages: severe time and logistical restrictions; the confounding influence of the "microlesion" effect, in which brain circuits are perturbed by edema around recently inserted leads $;{ }^{18}$ increased infection risk $;{ }^{5}$ and the inability to assess the chronic effects of therapies or to prototype strategies for chronic closed-loop control (automated adjustment of DBS stimulation parameters based on brain signals).

To address these shortcomings, we launched an investigational protocol utilizing a first-generation, totally implantable, "bidirectional neural interface," a prosthesis that delivers clinically indicated stimulation therapy and senses and stores brain activity. This device, Activa $\mathrm{PC}+\mathrm{S}$ (Medtronic Inc.), allows chronic, intermittent collection of field potential data over years, and this collection can be initiated by investigators in the clinic, by patients in their homes, or by automated algorithms. ${ }^{1,4,30,35,36}$ The device is available to research groups worldwide under investigator-initiated protocols or under CE Marking in European countries. ${ }^{17,22,27,32,39,40,42}$ In our study, the stimulation and sensing pulse generator was attached to both a subcortical and cortical electrode array to allow multisite recordings for network-level analyses. Here, we describe our initial experience with the device in 5 PD patients for over 1 year, focusing on the implantation technique, complications, recording capabilities, technical limitations, and solutions for researchers to mitigate these limitations.

\section{Methods \\ Patients}

Inclusion criteria for this study were a diagnosis of idiopathic PD by a movement disorders neurologist and clinical justification for DBS surgery based on the presence of motor fluctuations or medication-induced dyskinesia after treatment with antiparkinsonian medications at maximal tolerable levels. Patients with prominent tremor were excluded.

Patients provided written consent in accordance with the Declaration of Helsinki and the University of California, San Francisco, institutional review board. The study was also reviewed and approved by the US Food and Drug Administration (FDA) under a physician-sponsored investigational device exemption. The study was registered with the ClinicalTrials.gov database (http://clinicaltrials. gov), and its registration no. is NCT01934296.

\section{Activa PC+S Recording Capabilities}

The functional capabilities of the Activa $\mathrm{PC}+\mathrm{S}$ system have been detailed in preclinical publications. 1,4,30,35,36 $^{2}$ Briefly, the device has 8-channel connectivity, but voltage time series can be recorded from only 2 bipolar channels simultaneously. Two additional channels can be selected for simultaneous calculation and storage of spectral power in prespecified bandwidths, but we did not systematically test that capability. When sampling 2 time series channels continuously at the maximum sampling rate of 800 $\mathrm{Hz}$, the maximum recording duration that can be stored, before one is required to download data to an external computer, is 8 minutes and 42 seconds. The Activa $\mathrm{PC}+\mathrm{S}$ device is identical in shape and size to the standard FDAapproved Activa PC device commonly used for therapeutic neurostimulation in movement disorders.

\section{Surgery}

Quadripolar cylindrical leads with a $1.5-\mathrm{mm}$ contact height and 0.5-mm intercontact spacing (model 3389-40, Medtronic Inc.) were implanted in the subthalamic nuclei (STNs), using techniques described previously. ${ }^{37}$ A quadripolar paddle-type lead with a $12-\mathrm{mm}^{2}$ exposed surface area and 1-cm intercontact distance (model 3587A25, Resume II, Medtronic Inc.) was implanted over the primary motor cortex on one side of the brain only, except in the first patient whose cortical lead was a cylindrical lead with a 3-mm contact height and 4-mm intercontact spacing (model 3391-40, Medtronic Inc.). The cylindrical cortical lead was used only until regulatory approval was granted for the investigational use of the Resume II paddle lead in the subdural space. The cortical lead was passed through the same 15-mm frontal bur hole used for the STN lead but was directed posteriorly in the subdural space under fluoroscopic guidance such that at least one contact covered the arm area of the primary motor cortex, on the medial aspect of the hand knob, ${ }^{45}$ approximately 3 $\mathrm{cm}$ from the midline. Adequate anatomical localization of the cortical electrode array was confirmed using intraoperative CT computationally merged to preoperative MRI in Framelink 5.0 (StealthStation, Medtronic Inc.). ${ }^{31}$ Functional localization of the cortical lead was verified by reversal of the N20 waveform of the somatosensory evoked potential. ${ }^{8}$

Subthalamic leads were secured to the skull using a standard lead anchoring device (Stimloc, Medtronic Inc.). On the brain side implanted with a cortical lead, the lead exited the bur hole in a bone trough underneath the Stimloc base ring and was secured to the skull with a titanium dogbone-shaped miniplate (Fig. 1). After removing the head frame and inducing general anesthesia, a 3-cm parietal incision was made, through which the free ends of the STN and cortical leads were accessed. These were attached to $40-\mathrm{cm}$ lead extenders specifically designed for use with the Activa PC+S generator (model 3708740, Medtronic Inc.). The extenders were tunneled to an infraclavicular incision and attached to the pulse generator/recording unit, which was placed in a pocket over the pectoralis muscle. If bilateral therapy was clinically indicated, a second STN electrode was implanted, attached to an Activa SC pulse generator (without sensing capability), and placed in an infraclavicular pocket contralateral to the Activa $\mathrm{PC}+\mathrm{S}$ device.

\section{Patient Visits}

For most patients, brain recordings were performed 1 day, 10 days, 3 weeks, 1 month, 2 months, 3 months, 6 

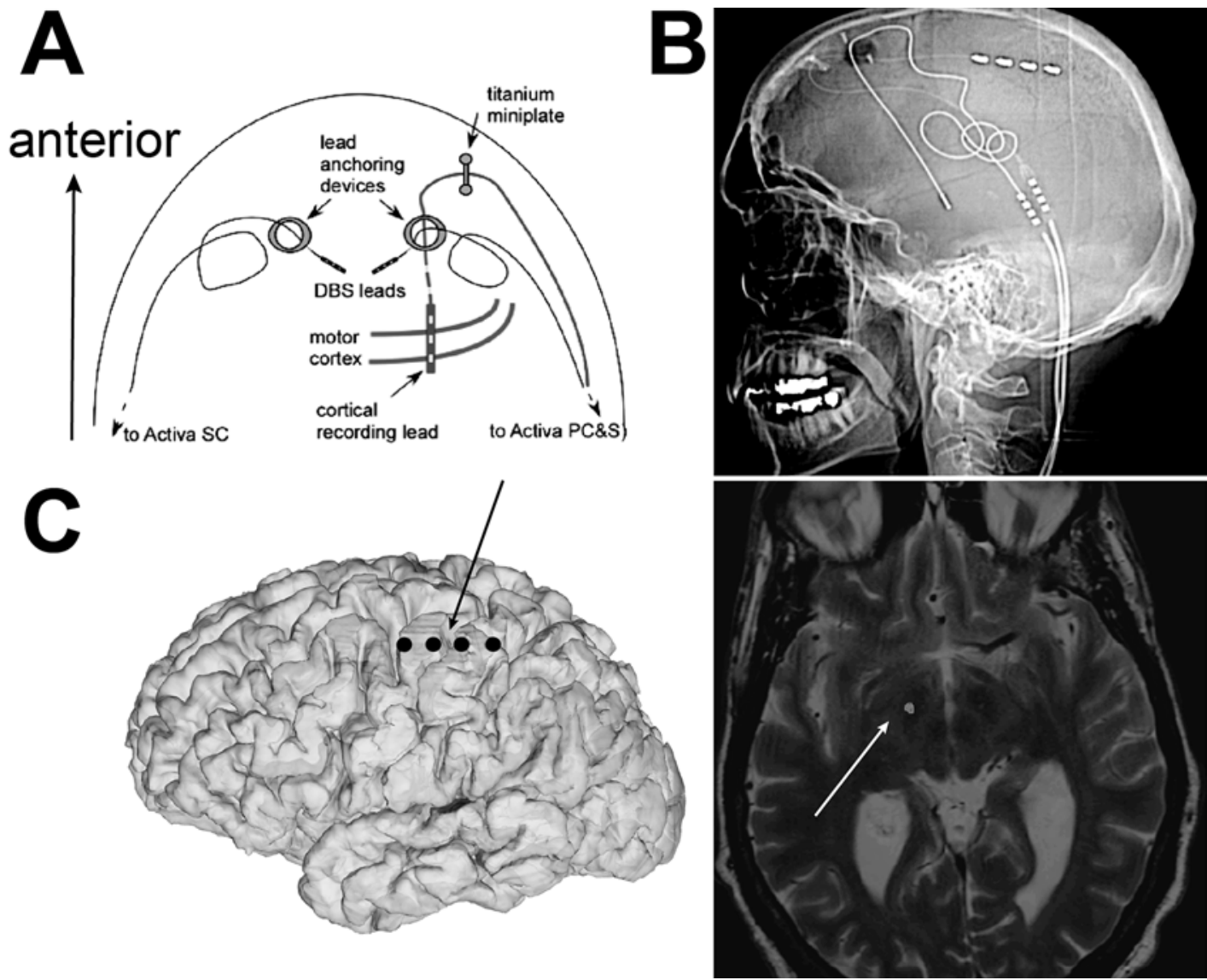

FIG. 1. A: Schematic of cranial hardware implantation (bilateral brain leads) as viewed from the top of the head. B: Lateral skull radiograph showing cranial hardware and proximal lead extenders. The patient in this case has unilateral DBS. C: Case 4.

Example lead locations. Black arrow indicates the central sulcus, and white arrow indicates the DBS electrode location in the STN. Electrode locations were determined by merging preoperative MRI to postoperative CT.

months, and 1 year after surgery. For the patient in Case 1 , the recordings at 1 day, 2 months, and 3 months were not performed. Subsequent modification of our protocol allowed the additional recordings in Cases 2-5. During research visits, brain recordings were performed on and off medications and/or with DBS on and off. Programming for chronic therapeutic DBS was initiated during the 1-month visit.

\section{Initiating Recordings and Data Downloading}

At study visits, recordings were triggered by placing the Sensing Programmer Telemetry Module (SPTM) on the patient's chest over the Activa $\mathrm{PC}+\mathrm{S}$ device. The Sensing Programmer (model 8181) was then used to initiate recordings, which either were set to last for a specified duration or were manually terminated. In addition to the recordings during formal study visits, patients could trigger brief recordings at home at any time by using the Medtronic Intercept patient programmer (model 37441). Study personnel had preprogrammed the recording duration for 1 minute for all home recordings. Data downloads were also performed with the Sensing Programmer and required at least 17-18 minutes (when memory was full).

\section{Recording Parameters}

We performed short-duration recordings $(<9$ minutes, repeated 1-3 times) at each research visit. Two channels of time series recordings were obtained simultaneously at $800 \mathrm{~Hz}$, the highest sampling rate available. We typically sampled one bipolar contact pair over the primary motor cortex and one bipolar contact pair in the STN. The selection of active cortical recording contacts was based on the pair showing the clearest somatosensory evoked potential reversal, the strongest movement-related high gamma response, ${ }^{6}$ and/or the strongest beta $(13-30 \mathrm{~Hz})$ peak in the power spectrum, at the time of initial surgical insertion. When these criteria were not in agreement, the intraoperative CT merged to the preoperative MRI was used to help select an optimal contact pair based on closest proximity to the posterior precentral gyrus. Additional recordings with other contacts were sometimes performed, particularly if more than one contact pair had shown strong movementrelated signals as described above. The selection criteria for the STN recording configuration differed before and after therapeutic DBS was activated. Prior to stimulation, we selected the bipolar pair whose LFP power spectrum showed the largest beta peak the day of or the day after surgery, off antiparkinsonian medications. If selection was ambiguous, the center contacts (1-2) were used. After 
initiating chronic therapeutic stimulation at 1 month, the contacts on either side of the stimulation contact(s), when available, were used to minimize artifacts. Since contacts used for stimulation could not simultaneously be used for recording, it is likely that the recording contacts used during chronic stimulation did not include the optimal therapeutic stimulation location within the STN. For all recordings, however, at least one of the recording contacts was in the motor STN or at the border of motor and nonmotor territories, based on intraoperative microelectrode recording.

\section{Behavioral States}

At each research visit and in each medication or stimulation state, brain recordings were performed with the patient in several different behavioral states, including at rest, while walking, and during an iPad reaching task, which has been previously described. ${ }^{10,29}$ Recording duration was 1-2 minutes for rest and walking and up to 6 minutes for the iPad task.

\section{Assessing Signal Stability}

To assess longitudinal signal stability, for each study visit, we calculated the root mean square (RMS) voltage for both the STN LFPs and motor cortex ECoG potentials. For this assessment, we used only resting-state recordings collected in the clinic, when patients were off both DBS and medications (except for the patient in Case 1, who had great difficulty tolerating the off-medication state and so maintained her regular medication regimen at her 10-day, 6-month, and 1-year visits). If more than one recording met these specifications for each visit, the RMS was calculated for each recording separately and then averaged across recordings to obtain 1 value per brain region and per patient at each time point. For STN recordings, we also omitted files contaminated by electrocardiogram (EKG) artifact (described below). ${ }^{27}$

We also assessed signal stability by calculating both beta and gamma power for motor cortex ECoG potentials and STN LFPs over time. We calculated power spectral density (PSD) using the Welch method in MATLAB (pwelch, $512 \mathrm{~ms}$ window, 1024 FFT length, and no overlap). For beta, we averaged the log PSD between 13 and $30 \mathrm{~Hz}$. For gamma, we averaged the log PSD in the broadband (high) gamma range $(70-100 \mathrm{~Hz})$, avoiding potential line noise at $60 \mathrm{~Hz}$. We calculated the height of the beta peak normalized to baseline power using a previously described method. ${ }^{43}$ Sometimes this local maximum was small and not readily distinguishable from baseline power. Therefore, data were also visually inspected to determine a threshold for reliable detection. In recording sessions in which multiple resting state data recordings were available, the PSD (for beta and gamma individually) or beta peak height was calculated for each recording, and the values were averaged across recordings. This analysis included the same recordings as the RMS analysis described above.

\section{Reaching Task}

Recording Paradigm

Twenty trials of the reaching task were performed for each session. Each trial included at least 5 seconds of rest or movement preparation and then 2 seconds of continuous movement. ${ }^{10,29}$ During the reaching task, electromyography and accelerometry activity was recorded with an external recording system to determine movement onsets (ActiveTwo, BioSemi). Synchronizing the iPad to the external recording system has been described previously. ${ }^{10,29}$ To synchronize the external recording system to the Activa $\mathrm{PC}+\mathrm{S}$ device, surface electrical stimulation was used to deliver 0.2-msec periodic pulses. Conductive pads were placed on the patient's head (near the forehead, but taking care to avoid large muscle groups to prevent uncomfortable muscle contractions during stimulation) and on the chest, near the implanted pulse generator (IPG). A train of pulses (approximately $10 \mathrm{~mA}$ ) was then delivered at both the beginning and end of the task. During the train of pulses, the frequency of the pulse train was changed (from 1 to $2 \mathrm{~Hz}$ and then back to $1 \mathrm{~Hz}$ or vice versa). This improved the ability to synchronize the pulse artifacts from both recording systems since occasional pulses were missed due to the short pulse width.

\section{Analysis}

We analyzed all recordings during the iPad reaching task to determine the presence of canonical movementrelated activity: beta amplitude decreases and gamma amplitude increases. ${ }^{4,6,7,20}$ Raw signals were visually inspected, and trials contaminated by artifact were excluded. Raw data were then filtered between 13 and $30 \mathrm{~Hz}$ for beta and 70-100 Hz for gamma, using a 2-way FIR1 filter (eegfilt from the eeglab ${ }^{11}$ toolbox, with firl parameters). The Hilbert transform of the filtered signal was then calculated, and the absolute value of this transform was obtained to derive the analytical amplitude over time in each frequency range. Spectrograms were also generated for visualization using the same approach but filtering all frequencies from 5 to 250 with a $2-\mathrm{Hz}$ bandwidth.

Changes during movement were determined by aligning data from all trials relative to the time of movement onset (time 0) and averaging across trials. The averaged amplitude was then normalized by a $500-\mathrm{msec}$ baseline prior to movement onset $(-2500$ to $-2000 \mathrm{msec}$ relative to movement). Data were normalized by subtracting the average baseline amplitude and dividing by the baseline standard deviation. This z-score procedure was performed separately for each frequency.

Recordings were categorized as having a detectable beta change if the z-scored values in the beta range (13-30 $\mathrm{Hz}$ ) were at or below -1.96 (corresponding to $\mathrm{p}<0.05$, uncorrected) for more than $150 \mathrm{msec}$ during the time period from $500 \mathrm{msec}$ before to 1 second after movement initiation. This is a relatively liberal threshold (with no correction for multiple comparisons) since we were not trying to determine the effect of movement on electrophysiology (which is already well established ${ }^{6,720}$ ) but were instead attempting to investigate the fidelity of the detection of this activity with the Activa $\mathrm{PC}+\mathrm{S}$ system.

An analogous procedure was performed for broadband gamma (70-100 Hz), except that the threshold was set to a $\mathrm{z}$-score value at or above 1.96 (since gamma increases are expected during movement). To avoid incorrectly catego- 
TABLE 1. Summary of characteristics of patients with PD

\begin{tabular}{cccccc}
\hline $\begin{array}{c}\text { Case } \\
\text { No. }\end{array}$ & Sex & $\begin{array}{c}\text { Age } \\
\text { (yrs) }\end{array}$ & $\begin{array}{c}\text { Disease } \\
\text { Duration } \\
\text { (yrs) }\end{array}$ & $\begin{array}{c}\text { Research } \\
\text { Hemisphere }\end{array}$ & $\begin{array}{c}\text { Baseline UPDRS } \\
\text { (off/on antiparkinsonian } \\
\text { medications) }\end{array}$ \\
\hline 1 & F & 47 & 15 & $\mathrm{Lt}$ & $68 / 16$ \\
\hline 2 & M & 62 & 8 & $\mathrm{Rt}$ & $30 / 14$ \\
\hline $3^{*}$ & $\mathrm{~F}$ & 56 & 4 & $\mathrm{Lt}$ & $46 / 35$ \\
\hline 4 & $\mathrm{M}$ & 59 & 7 & $\mathrm{Lt}$ & $29 / 14$ \\
\hline 5 & $\mathrm{M}$ & 60 & 14 & $\mathrm{Rt}$ & $44 / 31$ \\
\hline
\end{tabular}

rizing cases in which noise in the signal caused increases across all frequencies as true movement-related broadband gamma increases, a recording was only considered to have a reliable high gamma increase if there was also a beta power decrease.

\section{Results \\ Subjects}

Five patients, 2 female and 3 male, were enrolled in the study and received the implanted device. Patients were 47-62 years old at the time of surgery and had had PD between 4 and 15 years (Table 1). Four of the 5 patients received bilateral DBS therapy, with the "non-research" side implanted with a quadripolar STN lead attached to an ipsilateral Activa SC single-channel generator.

\section{Clinical Outcomes and Adverse Events}

Four of the 5 patients had improved motor function with DBS 1 year postoperatively, as indicated by reduced Unified Parkinson's Disease Rating Scale (UPDRS) Part III scores in the off-medication state (Table 2). One patient with minimal UPDRS improvement experienced a rapid progression in symptoms postimplantation, and subsequently developed features consistent with multisystem atrophy, parkinsonian subtype (MSA-P). Another patient, who had undergone unilateral implantation, experienced ipsilateral disease progression but did have a clinical benefit from DBS on the treated side. No patients reported any suicidal ideation during the study.

There were no serious adverse events related to the study protocol. Minor complications included the development of a painless, nonerythematous subgaleal fluid collection near the bur hole on the side implanted with the cortical lead, presumed to be CSF, which was self limited; thinning of the skin over the Activa $\mathrm{PC}+\mathrm{S}$ battery implantation, which remained stable without the need for surgical revision; and an episode of lightheadedness during programming, which resolved with rest. Each of these events occurred only once. Two protocol violations occurred: the inadvertent performance of postoperative MRI in one patient, without clinical sequelae, and the inadvertent activation of cortical stimulation for 30-45 minutes, without clinical sequelae, in another patient.

\section{Contact Localization}

Cortical electrode arrays were successfully placed over
TABLE 2. Patient outcomes 1 year after implantation of Activa PC+S

\begin{tabular}{ccccc}
\hline $\begin{array}{c}\text { Baseline } \\
\text { UPDe } \\
\text { No. } \\
\text { Off }\end{array}$ & $\begin{array}{c}\text { 1-Yr Postop } \\
\text { UPDRS, Off } \\
\text { Medications }\end{array}$ & $\begin{array}{c}\text { 1-Yr Postop } \\
\text { Off DBS }\end{array}$ & $\begin{array}{c}\text { Improvement 1 } \\
\text { Medications, } \\
\text { On DBS }\end{array}$ & $\begin{array}{c}\text { Mr Postop, Off } \\
\text { Medications, } \\
\text { On DBS* }\end{array}$ \\
\hline 1 & $16 \dagger$ & $8 \dagger$ & $5 \dagger$ & $68 \% \dagger$ \\
\hline 2 & 30 & 22 & 16 & $47 \%$ \\
\hline 3 & 46 & 51 & 50 & $9 \%$ \\
\hline $4 \ddagger$ & 29 & 52 & 41 & $-41 \%$ \\
\hline 5 & 44 & 38 & 22 & $50 \%$ \\
\hline
\end{tabular}

* Relative to baseline off medications.

$\dagger$ Patient tested on medications.

$\ddagger$ Unilateral implantation.

the left ( 3 patients) and right ( 2 patients) motor cortex and in the ipsilateral STN in all patients. See Fig. 1 for example lead locations and Table 3 for anterior commissure-posterior commissure coordinates for cortical contacts and the STN lead tip, measured from intraoperative CT fused to preoperative MRI. Comparing intraoperative to postoperative CT (acquired between 3 weeks to 3 months after surgery) revealed minimal migration of the cortical electrode array, with less than $3.5 \mathrm{~mm}$ of movement in each case (mean $1.7 \mathrm{~mm}$, range $0-3.5 \mathrm{~mm}$ ).

\section{Signal Quality and Stability Over 1 Year}

The quality of the recordings from the Activa $\mathrm{PC}+\mathrm{S}$ device with the patient off stimulation a few hours after implantation was similar to the quality of the intraoperative recordings obtained with an FDA-approved external sys-

TABLE 3. Electrocorticography contact and DBS electrode tip coordinates

\begin{tabular}{cccccc}
\hline $\begin{array}{c}\text { Case } \\
\text { No. }\end{array}$ & $\begin{array}{c}\text { Contact } \\
3\end{array}$ & $\begin{array}{c}\text { Contact } \\
2\end{array}$ & $\begin{array}{c}\text { Contact } \\
1\end{array}$ & $\begin{array}{c}\text { Contact } \\
0\end{array}$ & $\begin{array}{c}\text { DBS } \\
\text { Electrode Tip }\end{array}$ \\
\hline 1 & -26.24, & -25.99, & -25.52, & -25.05, & -10.54, \\
& -9.61, & -16.79, & -23.35, & -30.15, & -2.73, \\
& 63.07 & 64.24 & 64.24 & 64.24 & -4.87 \\
\hline 2 & 30.40, & 29.23, & 29.23, & 29.23, & 11.31, \\
& -7.63, & -17.86, & -27.87, & -38.20, & -2.35, \\
& 65.36 & 66.22 & 65.79 & 65.47 & -4.44 \\
\hline 3 & -27.52, & -26.35, & -26.74, & -26.94, & -11.39, \\
& -4.71, & -15.06, & -25.22, & -35.18, & 0.23, \\
& 67.15 & 67.15 & 67.15 & 67.15 & -4.04 \\
\hline 4 & -38.02, & -38.02, & -38.02, & -38.02, & -12.56, \\
& -6.59, & -16.55, & -27.39, & -37.94, & -2.25, \\
& 64.21 & 63.63 & 62.75 & 62.46 & -5.96 \\
\hline 5 & 28.43, & 28.43, & 28.43, & 28.43, & 12.50, \\
& 5.02, & -4.68, & -14.90, & -25.95, & -0.97, \\
& 63.59 & 65.94 & 67.61 & 69.62 & -5.51 \\
\hline
\end{tabular}

Coordinates presented as distance from the midpoint of the line connecting the anterior and posterior commissures, expressed in $\mathrm{mm}$. In each box the top number is the lateral coordinate; the middle number, the anteroposterior coordinate; and the lower number, the vertical coordinate. The positive direction is defined as right, anterior, and superior. 


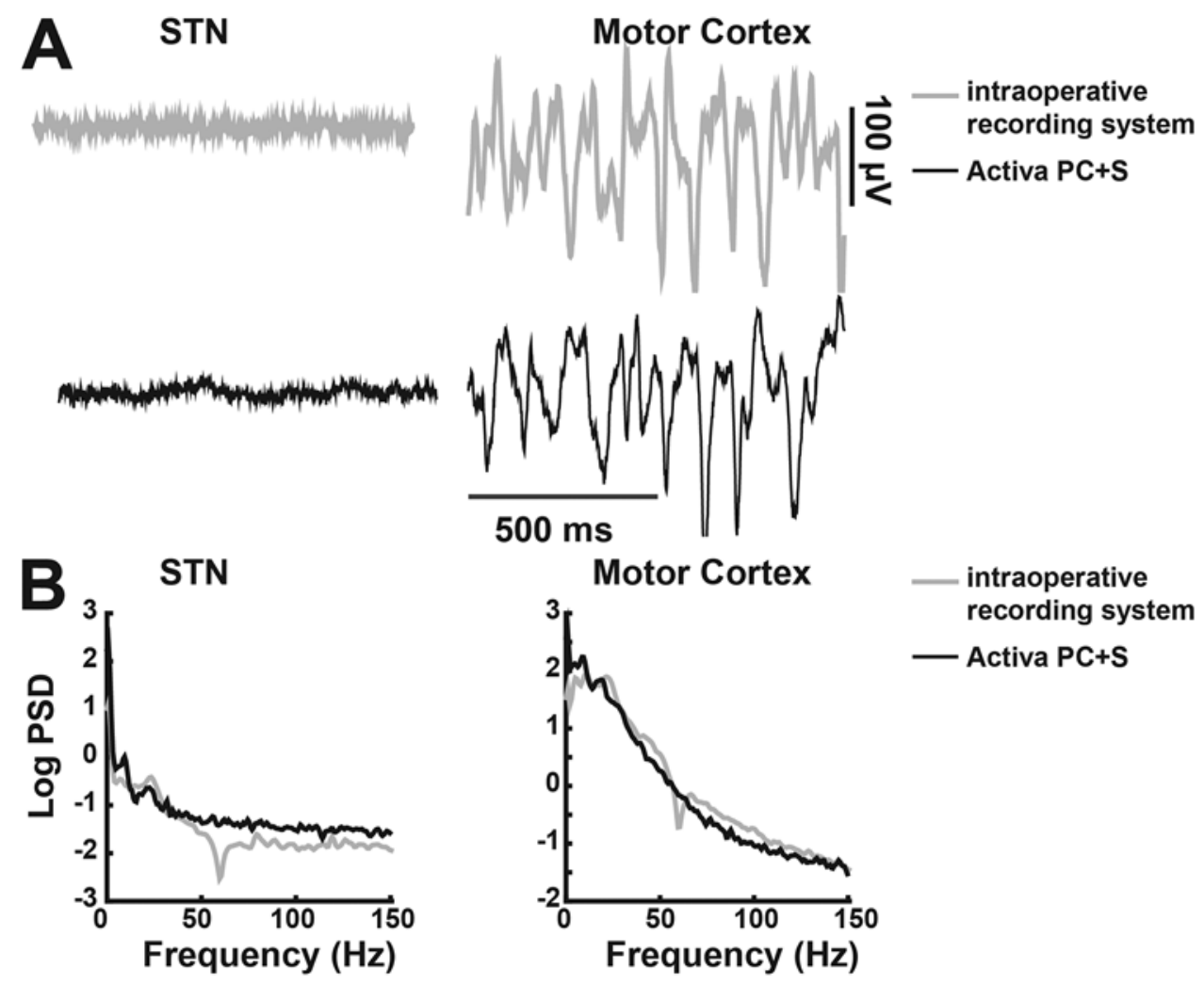

FIG. 2. Example of motor cortex ECoG potentials and STN LFPs raw data (A) and their power spectra (B) from the Activa PC+S system versus an external recording system designed for intraoperative electrophysiology (MicroGuide). Both recordings were obtained on the same day, a few hours apart, from the patient in Case 5. Note that notch filtering was used to remove line noise at $60 \mathrm{~Hz}$ in the intraoperative data. Line noise was not present in the Activa PC+S data.

tem designed for human electrophysiology (MicroGuide, Alpha Omega; Fig. 2), with the exception of some artifacts discussed below. This comparison has also been made in nonhuman primates. ${ }^{4}$ Subthalamic nucleus LFP amplitudes were lower than those of simultaneously recorded motor ECoG potentials ${ }^{9}$ (Fig. 3A). The average RMS voltage at rest, off DBS was $5.1 \pm 0.38 \mu \mathrm{V}$ (mean \pm standard deviation) for STN and $20.4 \pm 2.79 \mu \mathrm{V}$ for cortex. These RMS values remained relatively stable over time (Fig. 3B).

Electrode impedances for both cortical and STN leads increased over time (Fig. 4E). This increase occurred mainly in the first months following surgery. An initial impedance increase followed by long-term stabilization has also been observed for other implanted neural prostheses. ${ }^{33}$

\section{Detection of Physiologically Important Frequency Bands}

Prominent oscillatory activity in the beta band (13-30 $\mathrm{Hz}$ ) is expected in field potential recordings from structures in the motor system. Gamma band activity in the cortex, over a broad frequency range $(50-200 \mathrm{~Hz})$, is a useful marker of local cortical activation. ${ }^{19} \mathrm{~A}$ major goal of this study was to document the ability to record these canonical physiological signatures using the novel Activa $\mathrm{PC}+\mathrm{S}$ device. Log beta power (Fig. 4A) and log gamma power (Fig. 4B) at rest while off DBS varied between patients but were relatively stable over time within subjects, similar to observations in nonhuman primates. ${ }^{4}$ The amplitude of the PSD beta peak with respect to baseline power (see Methods) was more variable, but still there was not a systematic change in beta peak height over time (Fig. 4C-D). Consistent with the experience of other users of Activa $\mathrm{PC}+\mathrm{S},{ }^{22}$ an $\mathrm{STN}$ beta peak was not always clearly detected. Visual inspection suggested that any beta peak values with a height $<0.25 \log _{10}\left(\mu \mathrm{V}^{2} / \mathrm{Hz}\right)$ were difficult to distinguish from the rest of the spectrum and probably unreliable (Fig. 4F). Thirteen (31\%) of 42 recordings in the STN and $5(8 \%)$ of 62 recordings in the motor cortex failed to show a beta peak by this criterion (Table 4).

Variable beta peak detection may be unrelated to Activa $\mathrm{PC}+\mathrm{S}$ recording capabilities. Beta oscillatory activity is known to dynamically change with behavior including movement and cognition, 7,26 and in PD it is also affected by the medication state. ${ }^{15}$ Of note, our patient with the lowest rate of beta peak detection (Case 3) was eventually diagnosed with multisystem atrophy.

\section{Movement-Related Changes}

Examples of time-frequency plots showing movementrelated changes in motor cortex ECoG potentials during the iPad-based arm movement task are provided in Fig. 5. For all such recordings, we classified movement-related beta power decreases and gamma power increases as present or absent (see Methods). Almost all motor cortex recordings (54 [98\%] of 55) had a beta power decrease at movement initiation, and most exhibited a gamma power increase (37 [67\%] of 55). Since movement-related corti- 

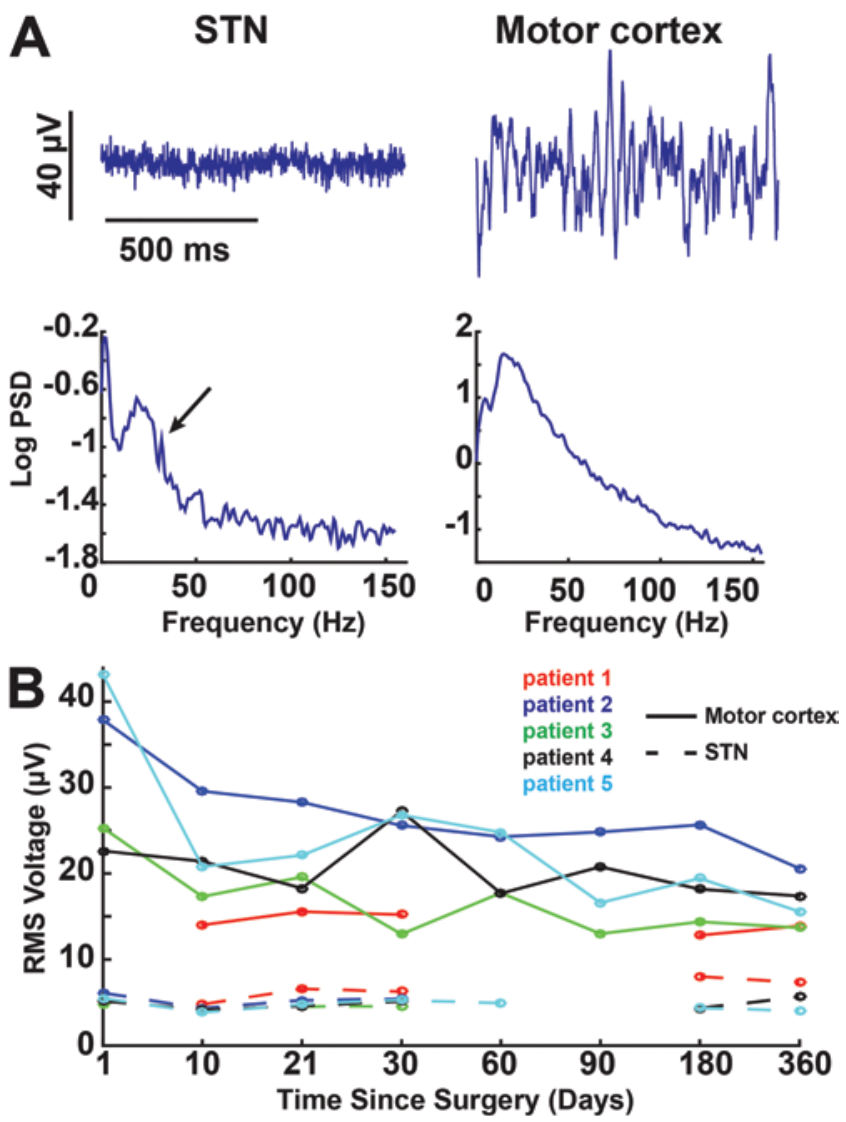

FIG. 3. Signal quality and evolution over time. A: Example signals and their power spectra from the patient in Case 2, 10 days postoperatively, for both STN and motor cortex. For the STN PSD, the sharp peak at 32 $\mathrm{Hz}$ (arrow), on the right shoulder of the beta peak, is artifactual (further elaborated in Fig. 6). B: Evolution of RMS voltage over time for all patients. Data for the patient in Case 1 at 1 day, 2 months, and 3 months are missing because the initial protocol did not include research visits at these time points. The STN recordings contaminated by EKG artifact (see Fig. 6) were also excluded.

cal gamma changes were reliably detected intraoperatively when the cortical paddle leads were connected to an external recording system, we suspect that the Activa $\mathrm{PC}+\mathrm{S}$ noise floor at high frequencies may have partially obscured movement-related gamma changes (Fig. 5C). Beta and gamma power changes were more difficult to detect in the STN than in cortex (Table 5). Note, though, that movement-related gamma is not always detected in the STN using intraoperative recording systems.

The ability to detect beta and gamma band changes during therapeutic neurostimulation will be important for the eventual use of neural interfaces for feedback-controlled stimulation. During DBS, movement-related signals in the motor cortex were still detectable at a similar level: 34 (94\%) of 36 recordings had detectable beta and $25(69 \%)$ of 36 recordings had detectable gamma. In the STN, however, signal detection was much reduced: only 4 $(15 \%)$ of 26 recordings had detectable beta and $0(0 \%)$ of 26 recordings had detectable gamma (Table 5). We noted that the patient in Case 5 had very few cortical recordings with detectable movement-related gamma increases prior to stimulation initiation and had more such events detected during stimulation. It is possible that this change could reflect a stimulation-induced improvement in cortical activation, but this was only observed in one patient.

Accurate signal averaging of event-related neural activity over multiple repetitions of a task depends on correct event alignment. This depends on the reliability of the data sampling rate over successive trials. We examined the consistency of the Activa $\mathrm{PC}+\mathrm{S}$ sampling rate for all recordings with event-related activity (that is, during the iPad reaching task) by using the synchronization procedure described in Methods. We found that the sampling rate varied from 788 to $794 \mathrm{~Hz}$ in extreme cases and was typically between 792 and $794 \mathrm{~Hz}$, when the nominal sampling rate was programmed at $800 \mathrm{~Hz}$.

\section{Artifacts}

Brain sensing during stimulation is one of the most technically challenging aspects of totally implantable brain recording systems. During therapeutic stimulation, artifacts occurred at the stimulation frequency and also at its folded subharmonics (Fig. 6A). Stimulation artifacts were more prominent for the STN LFPs than the ECoG potentials, given the smaller signal amplitude as well as the proximity of the stimulation source to the recording array. For cortical recordings, detection of canonical movement-related changes in beta and gamma bands during DBS was similar to when DBS was off, whereas for STN recordings, DBS reduced the fidelity of detection of event-related changes (Table 5). In general, stimulation artifacts arise from 2 main sources: volume conduction through the brain and coupling between the stimulation and recording circuits with the stimulation/sensing device. While the former can be greatly reduced by signal detection at a distance from the stimulating electrode, the latter is more prominent than is the case for similar recordings from externalized leads connected to large external amplifiers ${ }^{10}$ for which isolation of stimulation and recording circuits is technically simpler.

Several narrowband artifacts were also present in the Activa $\mathrm{PC}+\mathrm{S}$ recordings even when stimulation was off. The frequency of these artifacts depended on the sampling rate used. For sampling rates of $800 \mathrm{~Hz}$, the most obvious of these artifacts occurred at 200 and $32 \mathrm{~Hz}$ (Fig. 6B). The origin of the $200-\mathrm{Hz}$ artifact (based on communications from Medtronic engineers) is an internal firmware processing step. The $32-\mathrm{Hz}$ artifact is attributable to an internal Activa PC+S clock. Avoiding analysis of these frequencies can prevent the misinterpretation of data. This is straightforward for the $200-\mathrm{Hz}$ artifact, but is more difficult for the $32-\mathrm{Hz}$ artifact since it is close to the beta band, an important frequency band in the motor system and in movement disorders. ${ }^{7,12}$ When signal amplitude is low, this artifact could mimic or obscure a physiological beta signal (Figs. 3A, 4F, and 6B). High-amplitude signals, like those recorded from motor cortex or STN recordings with a robust LFP (Fig. 4F), are not strongly influenced.

Subthalamic nucleus recordings from Contact 0 (the most ventral contact) usually had EKG contamination (Fig. 6C). This is believed to originate from current leakage into the IPG at the insertion site of the device lead 

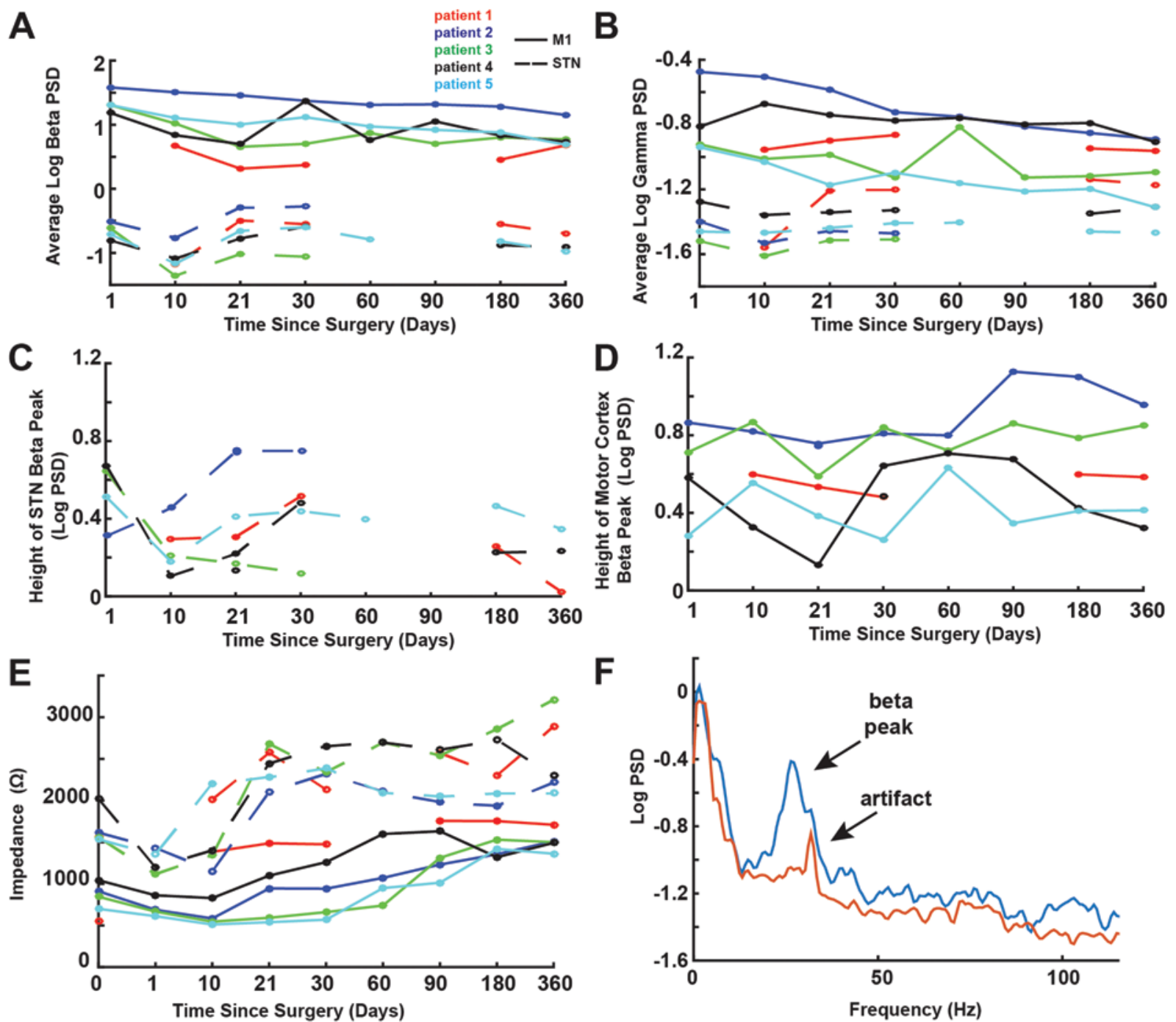

FIG. 4. Power and impedance change over time. A: Average log beta PSD over time for all subjects. B: Average log broadband gamma over time for all subjects. C: Average log beta peak height in the STN over time for all subjects. Data for the patient in Case 1 at 1 day, 2 months, and 3 months are missing because the initial protocol did not include research visits at these time points. Moreover, the STN recordings contaminated by EKG artifact (see Fig. 6) were excluded. D: Average log beta peak height in the motor cortex over time for all subjects. E: Average monopolar impedances averaged across all electrode contacts for each region (motor cortex and STN). Impedances gradually increase over time. F: Example of an STN LFP power spectrum with (Case 4, 1 day after surgery) and without (Case 4, 10 days after surgery) the expected broad peak in the alpha-beta range. Note that the sharp peak at $32 \mathrm{~Hz}$ from the 10-day postsurgery recording is an artifact (see Results and Fig. 6).

TABLE 4. Recordings (at rest and off medications) with detected beta peaks in the LFP or ECoG power spectrum $\left(>0.25 \log _{10}[\mu \mathrm{V} 2 \mid\right.$ $\mathrm{Hz}])$

\begin{tabular}{ccc}
\hline \multirow{2}{*}{$\begin{array}{c}\text { Case } \\
\text { No. }\end{array}$} & \multicolumn{2}{c}{ Detected/Total } \\
\cline { 2 - 3 } & Beta Detected in STN & Beta Detected in Motor Cortex \\
\hline $1^{*}$ & $4 / 5(80 \%)$ & $5 / 5(100 \%)$ \\
\hline 2 & $5 / 5(100 \%)$ & $10 / 10(100 \%)$ \\
\hline 3 & $2 / 7(28 \%)$ & $14 / 14(100 \%)$ \\
\hline 4 & $4 / 10(40 \%)$ & $13 / 15(87 \%)$ \\
\hline 5 & $14 / 15(93 \%)$ & $15 / 18(83 \%)$ \\
\hline Total & $29 / 42(69 \%)$ & $57 / 62(92 \%)$ \\
\hline
\end{tabular}

* On-medication data are included for Case 1. extender over the pectoralis muscle. Applying a sealant at the junction between lead extender and pulse generator may reduce the EKG artifact. ${ }^{27}$ We also observed baseline deflections in the signal at the initiation of recordings. This channel startup transient decayed back to $0 \mathrm{~V}$ over 3-10 seconds (Fig. 6D). Sudden "jumps" in voltage were another type of transient baseline deflection observed, followed by decay back to 0 (Fig. 6E). These occurred infrequently. Of note, $60-\mathrm{Hz}$ line noise and its harmonics were not prominent, as is expected based on the isolation of a totally implantable device from external sources of noise.

\section{Discussion}

We used a novel, investigational, totally implantable, 


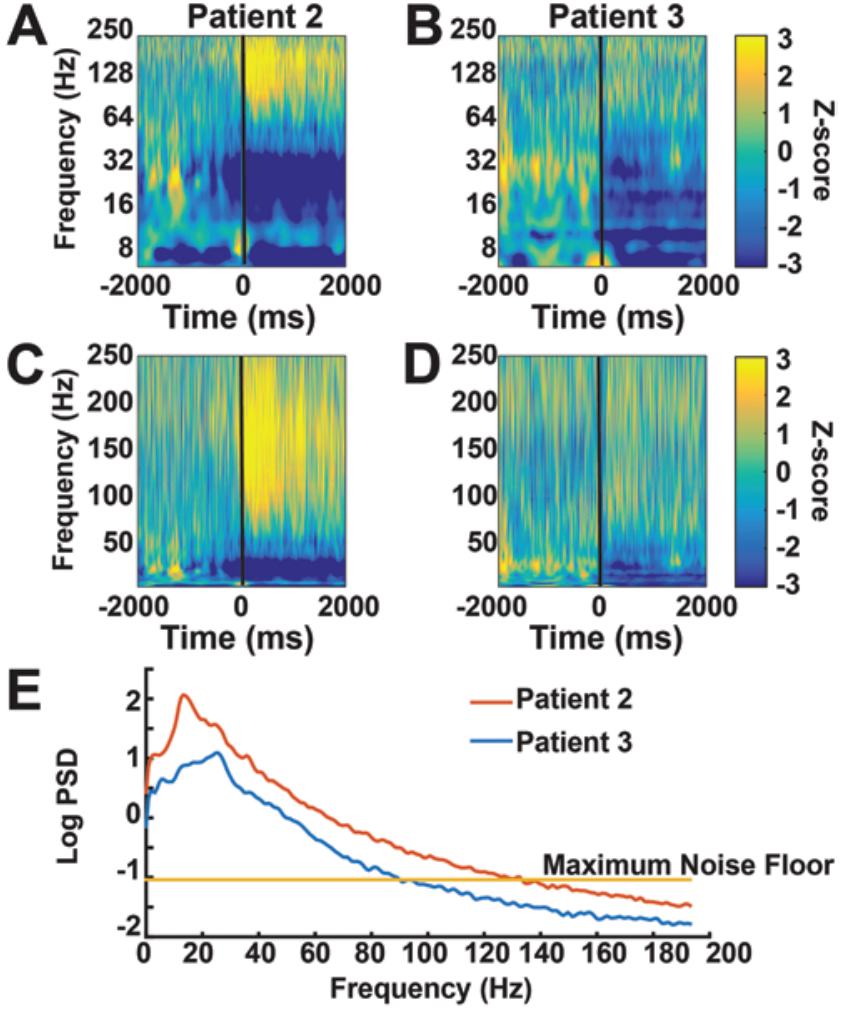

FIG. 5. Detection of movement-related changes in motor cortex ECoG potentials and their relationship to the device noise floor. Movementrelated spectrograms (plotted on a log scale) aligned to movement onset (time 0 ) for a recording session that did (A, Case 2, 10 days postoperative) and did not (B, Case 3, 3 weeks postoperative) exhibit a movementrelated gamma increase. Both examples have a beta decrease. The same plots on a nonlog scale (C and D, respectively). The PSD plots (E) for recordings shown in panels A-D, as well as the maximum manufacturer-specified device noise floor. Note that gamma activity may be difficult to detect reliably in panels $B$ and $D$ because gamma activity in that recording is near the device noise floor.

bidirectional neural interface to record motor cortex ECoG potentials and STN LFPs in 5 PD patients over 1 year. There were no serious surgical complications, no unintended interruptions in DBS therapy, and no major malfunctions in the recording features of the device. Signal amplitudes in both cortex and STN (measured as both RMS voltage and detection of beta and gamma power) remained relatively stable despite an increase in average monopolar contact impedances over time in both regions. In motor cortex, the canonical movementrelated beta frequency decrease ${ }^{7}$ was reliably detected, the movement-related high gamma increase ${ }^{6}$ was seen in two-thirds of the recordings, and therapeutic stimulation did not preclude detection of these signals. The detection of movement-related changes in the STN was less reliable and more strongly influenced by stimulation artifacts. The STN LFP signal can, however, be improved by utilizing specific stimulation and recording configurations. These findings should be of use to investigators planning research protocols using Activa $\mathrm{PC}+\mathrm{S}$, as well as device companies designing the next generation of bidirectional neural interfaces.
TABLE 5. Recordings with movement-related changes in beta and gamma frequencies

\begin{tabular}{|c|c|c|c|c|}
\hline \multirow{3}{*}{$\begin{array}{l}\text { Case } \\
\text { No. }\end{array}$} & \multicolumn{4}{|c|}{$\begin{array}{l}\text { No. of Recordings w/ Detected Changes/ } \\
\text { Total No. of Recordings }\end{array}$} \\
\hline & \multicolumn{2}{|c|}{ Beta Frequencies } & \multicolumn{2}{|c|}{ Gamma Frequencies } \\
\hline & STN & Motor Cortex & STN & Motor Cortex \\
\hline \multicolumn{5}{|c|}{ Off DBS } \\
\hline 1 & $2 / 5(40 \%)$ & $8 / 9(89 \%)$ & $0 / 5(0 \%)$ & $6 / 9(67 \%)$ \\
\hline 2 & $11 / 12(92 \%)$ & $12 / 12(100 \%)$ & $5 / 12(42 \%)$ & $12 / 12(100 \%)$ \\
\hline 3 & $4 / 11(36 \%)$ & $11 / 11(100 \%)$ & $1 / 11(9 \%)$ & $5 / 11(45 \%)$ \\
\hline 4 & $8 / 11(72 \%)$ & $12 / 12(100 \%)$ & $4 / 11(36 \%)$ & $12 / 12(100 \%)$ \\
\hline 5 & $9 / 10(90 \%)$ & $11 / 11(100 \%)$ & $3 / 10(30 \%)$ & $2 / 11(18 \%)$ \\
\hline Total & $34 / 49(69 \%)$ & $54 / 55(98 \%)$ & $13 / 49(26 \%)$ & $37 / 55(67 \%)$ \\
\hline \multicolumn{5}{|c|}{ On DBS } \\
\hline 1 & $2 / 5(40 \%)$ & $3 / 5(60 \%)$ & $0 / 5(0 \%)$ & $3 / 5(60 \%)$ \\
\hline 2 & $1 / 9(0 \%)$ & $9 / 9(100 \%)$ & $0 / 9(0 \%)$ & $9 / 9(100 \%)$ \\
\hline 3 & $1 / 6(17 \%)$ & $6 / 6(100 \%)$ & $0 / 6(0 \%)$ & $1 / 6(17 \%)$ \\
\hline 4 & $0 / 6(0 \%)$ & $8 / 8(100 \%)$ & $0 / 6(0 \%)$ & $8 / 8(100 \%)$ \\
\hline 5 & $N A^{*}$ & $8 / 8(100 \%)$ & $N A^{*}$ & $4 / 8(50 \%)$ \\
\hline Total & $4 / 26(15 \%)$ & $34 / 36(94 \%)$ & $0 / 26(0 \%)$ & $25 / 36(69 \%)$ \\
\hline
\end{tabular}

\section{Neural Interfaces for Invasive Recording in Humans}

Activa $\mathrm{PC}+\mathrm{S}$ is one of a few chronic, invasive recording devices available for human use. Others are the RNS device (Neuropace Inc.), ${ }^{23,38}$ the first fully implanted responsive neurostimulation device approved for use in humans for epilepsy treatment, and the BrainGate device (BrainGate Co.), a brain-machine interface designed for patients with paralysis. ${ }^{14,41}$ While all 3 of these devices allow invasive electrophysiological recording, there are important differences, advantages, and limitations to each. Both the RNS and Activa PC+S devices are fully internalized devices, whereas the BrainGate device has an externalized component. The internalized devices have the significant advantage of a reduced risk of infection and the capacity for ambulatory recording outside of a specialized laboratory, at the expense of limited bandwidth. In contrast, the BrainGate system allows simultaneous recording from many more electrodes, as well as much higher sampling rates that permit detection of single-unit activity in addition to field potentials.

Both BrainGate and Activa $\mathrm{PC}+\mathrm{S}$ allow rapid, immediate streaming of data to external computers, facilitating brain-machine interface applications such as real-time control of a motor prosthesis ${ }^{14,41}$ or prototyping of feedback-controlled stimulation algorithms. ${ }^{17}$ Feedback control may improve DBS therapy by real-time adjustment of stimulation parameters in response to fluctuating signs and symptoms. ${ }^{16,28}$ Long-term studies utilizing the BrainGate and RNS devices indicate stable recordings for up to 5 years for both, based on direct inspection of the signals (BrainGate) or continued clinical efficacy (RNS), ${ }^{2,3,34}$ 


\section{A. Stimulation artifacts}

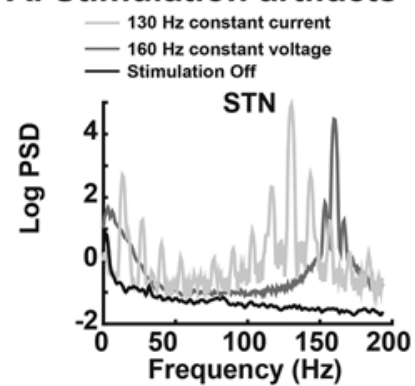

B. Narrowband artifacts

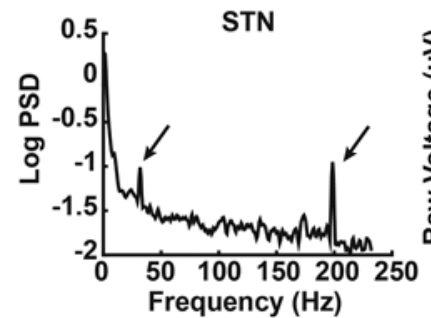

D. Baseline Deflection Artifact

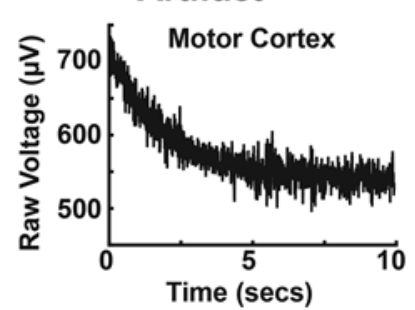

Time (secs)
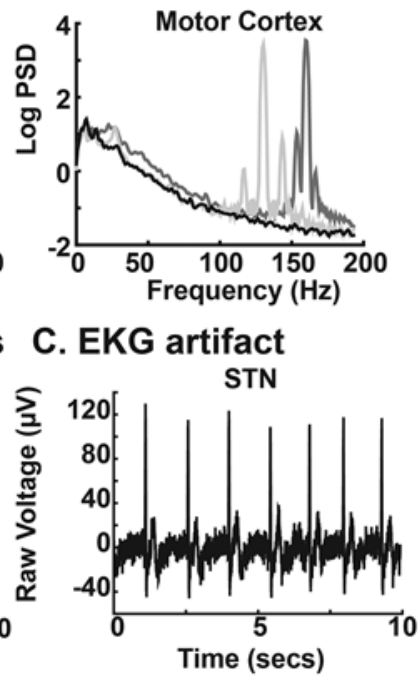

E. Putative Movement Artifacts

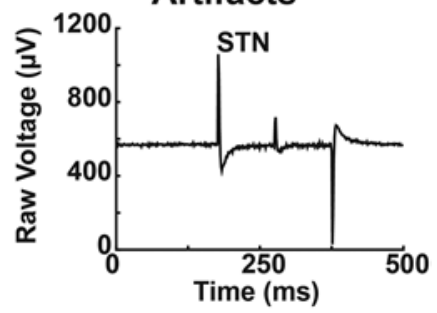

FIG. 6. Illustration of common Activa PC+S electrical artifacts. A: Example of DBS stimulation artifacts for constant current and constant voltage settings as well as off DBS in both motor cortex and STN in the same patient (Case 4). Stimulation parameters: c+1-, $160 \mathrm{~Hz}, 3 \mathrm{~V}, 60$ usec pulse width versus c+2-, $130 \mathrm{~Hz}, 4.9 \mathrm{~mA}, 90$ usec. Constant voltage recording occurred at 1 month postoperatively, and constant current and off-stimulation recordings occurred at 1 year postoperatively. Note that in all cases, the STN recordings were obtained from bipolar contact pairs bordering stimulation to minimize artifact $(0-2$ for constant voltage recording and 1-3 for constant current and off stimulation). Motor cortex recordings were obtained from contacts 9-11 in all recordings. B: Example of narrowband frequency artifacts (arrows) associated with intrinsic firmware or clock configuration properties in Active PC+S (see Results for more information). C: Example of EKG artifact in the STN. D: Example of baseline deflection artifact associated with a filter startup transient. This occurred at the beginning of each recording for both electrode contacts. E: Example of transient baseline deflections of unknown etiology. These deflections can occur in motor cortex or STN.

These findings support the idea that long-term stable electrophysiological recording in humans is possible.

Of the available implantable devices with a sensing capability, Activa $\mathrm{PC}+\mathrm{S}$ is the only one designed for continuous stimulation and thus is suitable to deliver therapy for any disorder treatable with open-loop DBS. ${ }^{21}$ The use of Activa $\mathrm{PC}+\mathrm{S}$ in humans with $\mathrm{PD}$ for single-site subcortical recording with continuous stimulation was recently reported..$^{22,27}$ Here, we describe the use of a multisite recording configuration (cortex and basal ganglia) via the Activa $\mathrm{PC}+\mathrm{S}$ device in humans. ${ }^{39}$

\section{Device Limitations and Mitigation Strategies}

Many of the technical limitations of Activa $\mathrm{PC}+\mathrm{S}$ are attributable to the constraints associated with integrating the sensing function into a small, totally implantable device. Canonical movement-related high gamma changes in motor cortex, reliably detected using ECoG arrays attached percutaneously to external recording systems, $, 6,7,20,29$ were not always apparent using Activa $\mathrm{PC}+\mathrm{S}$. Broadband gamma (also called "high gamma") activity tracks local cortical function and is thought to be a surrogate for local neural activity. ${ }^{19}$ The optimal frequencies for gamma power detection are above $70 \mathrm{~Hz}$, for which signal amplitudes are near or below the device noise floor of Activa $\mathrm{PC}+\mathrm{S}$ in some patients (Fig. 5B and D). Further, cross frequency interactions between the amplitude of high gamma and the phase of lower frequency rhythms may provide important control signals for adaptive (closed loop) stimulation, ${ }^{9,10}$ underscoring the importance of high gamma sensing.

The stimulation artifact is more pronounced with Activa $\mathrm{PC}+\mathrm{S}$ than when stimulating and recording from externalized leads. ${ }^{10}$ This is probably due to coupling of the stimulation and recording circuits within the pulse generator. The stimulation artifact is particularly challenging for STN because of its low signal amplitude and proximity to the site of stimulation. This may limit the feasibility of close-loop DBS approaches using STN LFP beta power as a control signal. ${ }^{16}$ Stimulation artifacts may be reduced by the use of the $100-\mathrm{Hz}$ low pass filter. The use of constant voltage rather than constant current stimulation aids in common mode rejection of stimulation artifacts. Stimulation frequency may be selected judiciously to align stimulation artifacts away from the frequency bands of interest (for example, $140-\mathrm{Hz}$ stimulation sampled at $422 \mathrm{~Hz}$ results in artifacts above $130 \mathrm{~Hz}$ or below $10 \mathrm{~Hz}$, away from the frequency bands of greatest interest for many applications). Recording from a bipolar configuration that brackets a monopolar stimulating contact is ideal for stimulation artifact reduction, but this may not be possible if the clinically optimal stimulation contact is on one end of the contact array. Finally, a calibration procedure can be performed prior to each recording to determine optimal STN gain. ${ }^{27}$

Fully implantable devices pose a trade-off between the recording capability of the device and the longevity of the power supply. Using the maximum available sampling rate of $800 \mathrm{~Hz}$ for 2-channel recording affords less than 9 minutes of data storage prior to download. Memory capacity in Activa $\mathrm{PC}+\mathrm{S}$ can be improved by lowering the sampling rate, recording only spectral power at a prespecified frequency instead of time domain signals, sampling only one channel, or using the data compression (which may reduce the signal/noise ratio and is not recommended when stimulation is on).

Characterizing the variability in the sampling rate was critical for the analysis of task-related activity since this variability affects precise event alignment when signal averaging over multiple trials. Without changing the nominal programmed sampling rate, the actual sampling rate can vary between patients and between recording sessions within a patient. To address this, we delivered synchronization pulses at the beginning and end of each task record- 
ing and calculated the sampling rate from the number of samples collected and the length of the interval between pulses. For longer recordings, additional synchronization pulses may be necessary.

\section{Conclusions}

We report our initial experience with subcortical neurostimulation combined with chronic cortical and subcortical recording for 1 year in 5 PD patients using a bidirectional neural interface. The acquisition of high-quality, long-term ECoG and LFP data is achievable. For most disorders, brain network abnormalities underlying disease expression are yet to be discovered. Totally implantable sensing devices hold promise both for signal discovery and for feedback-controlled stimulation. These developments will be facilitated by improvements in the signal/noise ratio, increased bandwidth and memory, reduced time for data downloading, and better methods of synchronizing internal data sampling with external monitors and computers.

\section{Acknowledgments}

We thank Andrew Miller for proofreading the manuscript and for his help with the cortical reconstruction shown in Fig. 1C.

This study was supported by the UC President's Postdoctoral fellowship (N.C.S.), the National Institutes of Health Neurological Disorders and Stroke Grant No. R01 NS090913-01 (P.A.S.), and the Michael J. Fox Foundation (C.D.H.).

\section{References}

1. Afshar P, Khambhati A, Stanslaski S, Carlson D, Jensen $\mathrm{R}$, Linde D, et al: A translational platform for prototyping closed-loop neuromodulation systems. Front Neural Circuits 6:117, 2013

2. Bacher D, Jarosiewicz B, Masse NY, Stavisky SD, Simeral JD, Newell K, et al: Neural point-and-click communication by a person with incomplete locked-in syndrome. Neurorehabil Neural Repair 29:462-471, 2015

3. Bergey GK, Morrell MJ, Mizrahi EM, Goldman A, King-Stephens D, Nair D, et al: Long-term treatment with responsive brain stimulation in adults with refractory partial seizures. Neurology 84:810-817, 2015

4. Connolly AT, Muralidharan A, Hendrix C, Johnson L, Gupta $\mathrm{R}$, Stanslaski S, et al: Local field potential recordings in a non-human primate model of Parkinsons disease using the Activa PC + S neurostimulator. J Neural Eng 12:066012, 2015

5. Constantoyannis C, Berk C, Honey CR, Mendez I, Brownstone RM: Reducing hardware-related complications of deep brain stimulation. Can J Neurol Sci 32:194-200, 2005

6. Crone NE, Miglioretti DL, Gordon B, Lesser RP: Functional mapping of human sensorimotor cortex with electrocorticographic spectral analysis. II. Event-related synchronization in the gamma band. Brain 121:2301-2315, 1998

7. Crone NE, Miglioretti DL, Gordon B, Sieracki JM, Wilson MT, Uematsu S, et al: Functional mapping of human sensorimotor cortex with electrocorticographic spectral analysis. I. Alpha and beta event-related desynchronization. Brain 121:2271-2299, 1998

8. Crowell AL, Ryapolova-Webb ES, Ostrem JL, Galifianakis NB, Shimamoto S, Lim DA, et al: Oscillations in sensorimotor cortex in movement disorders: an electrocorticography study. Brain 135:615-630, 2012

9. de Hemptinne C, Ryapolova-Webb ES, Air EL, Garcia PA,
Miller KJ, Ojemann JG, et al: Exaggerated phase-amplitude coupling in the primary motor cortex in Parkinson disease. Proc Natl Acad Sci US A 110:4780-4785, 2013

10. de Hemptinne C, Swann NC, Ostrem JL, Ryapolova-Webb ES, San Luciano M, Galifianakis NB, et al: Therapeutic deep brain stimulation reduces cortical phase-amplitude coupling in Parkinson's disease. Nat Neurosci 18:779-786, 2015

11. Delorme A, Makeig S: EEGLAB: an open source toolbox for analysis of single-trial EEG dynamics including independent component analysis. J Neurosci Methods 134:9-21, 2004

12. Hammond C, Bergman H, Brown P: Pathological synchronization in Parkinson's disease: networks, models and treatments. Trends Neurosci 30:357-364, 2007

13. Herrington TM, Cheng JJ, Eskandar EN: Mechanisms of deep brain stimulation. J Neurophysiol 115:19-38, 2016

14. Hochberg LR, Serruya MD, Friehs GM, Mukand JA, Saleh M, Caplan AH, et al: Neuronal ensemble control of prosthetic devices by a human with tetraplegia. Nature 442:164-171, 2006

15. Kühn AA, Kupsch A, Schneider GH, Brown P: Reduction in subthalamic $8-35 \mathrm{~Hz}$ oscillatory activity correlates with clinical improvement in Parkinson's disease. Eur J Neurosci 23:1956-1960, 2006

16. Little S, Pogosyan A, Neal S, Zavala B, Zrinzo L, Hariz M, et al: Adaptive deep brain stimulation in advanced Parkinson disease. Ann Neurol 74:449-457, 2013

17. Malekmohammadi M, Herron J, Velisar A, Blumenfeld Z, Trager MH, Chizeck HJ, et al: Kinematic adaptive deep brain stimulation for resting tremor in Parkinson's disease. Mov Disord 31:426-428, 2016

18. Mann JM, Foote KD, Garvan CW, Fernandez HH, Jacobson CE IV, Rodriguez RL, et al: Brain penetration effects of microelectrodes and DBS leads in STN or GPi. J Neurol Neurosurg Psychiatry 80:794-797, 2009

19. Manning JR, Jacobs J, Fried I, Kahana MJ: Broadband shifts in local field potential power spectra are correlated with single-neuron spiking in humans. J Neurosci 29:13613-13620, 2009

20. Miller KJ, Leuthardt EC, Schalk G, Rao RPN, Anderson NR, Moran DW, et al: Spectral changes in cortical surface potentials during motor movement. J Neurosci 27:2424-2432, 2007

21. Miocinovic S, Somayajula S, Chitnis S, Vitek JL: History, applications, and mechanisms of deep brain stimulation. JAMA Neurol 70:163-171, 2013

22. Neumann WJ, Staub F, Horn A, Schanda J, Mueller J, Schneider GH, et al: Deep brain recordings using an implanted pulse generator in Parkinson's disease. Neuromodulation 19:20-24, 2016

23. Okun MS, Foote KD, Wu SS, Ward HE, Bowers D, Rodriguez RL, et al: A trial of scheduled deep brain stimulation for Tourette syndrome: moving away from continuous deep brain stimulation paradigms. JAMA Neurol 70:85-94, 2013

24. Oswal A, Brown P, Litvak V: Synchronized neural oscillations and the pathophysiology of Parkinson's disease. Curr Opin Neurol 26:662-670, 2013

25. Panov F, Levin E, de Hemptinne C, Swann NC, Qasim S, Miocinovic S, et al: Intraoperative electrocorticography for physiological research in movement disorders: principles and experience in 200 cases. J Neurosurg 126:122-131, 2017

26. Pfurtscheller G: Central beta rhythm during sensorimotor activities in man. Electroencephalogr Clin Neurophysiol 51:253-264, 1981

27. Quinn EJ, Blumenfeld Z, Velisar A, Koop MM, Shreve LA, Trager MH, et al: Beta oscillations in freely moving Parkinson's subjects are attenuated during deep brain stimulation. Mov Disord 30:1750-1758, 2015

28. Rosin B, Slovik M, Mitelman R, Rivlin-Etzion M, Haber SN, Israel Z, et al: Closed-loop deep brain stimulation is superior in ameliorating parkinsonism. Neuron 72:370-384, 2011 
29. Rowland NC, De Hemptinne C, Swann NC, Qasim S, Miocinovic $\mathrm{S}$, Ostrem JL, et al: Task-related activity in sensorimotor cortex in Parkinson's disease and essential tremor: changes in beta and gamma bands. Front Hum Neurosci 9:512, 2015

30. Ryapolova-Webb E, Afshar P, Stanslaski S, Denison T, de Hemptinne C, Bankiewicz K, et al: Chronic cortical and electromyographic recordings from a fully implantable device: preclinical experience in a nonhuman primate. J Neural Eng 11:016009, 2014

31. Shahlaie K, Larson PS, Starr PA: Intraoperative computed tomography for deep brain stimulation surgery: technique and accuracy assessment. Neurosurgery 68 (1 Suppl Operative): $114-124,2011$

32. Shute JB, Okun MS, Opri E, Molina R, Rossi PJ, MartinezRamirez D, et al: Thalamocortical network activity enables chronic tic detection in humans with Tourette syndrome. Neuroimage Clin 12:165-172, 2016

33. Sillay KA, Rutecki P, Cicora K, Worrell G, Drazkowski $\mathrm{J}$, Shih JJ, et al: Long-term measurement of impedance in chronically implanted depth and subdural electrodes during responsive neurostimulation in humans. Brain Stimulat 6:718-726, 2013

34. Simeral JD, Kim SP, Black MJ, Donoghue JP, Hochberg LR: Neural control of cursor trajectory and click by a human with tetraplegia 1000 days after implant of an intracortical microelectrode array. J Neural Eng 8:025027, 2011

35. Stanslaski S, Afshar P, Cong P, Giftakis J, Stypulkowski P, Carlson D, et al: Design and validation of a fully implantable, chronic, closed-loop neuromodulation device with concurrent sensing and stimulation. IEEE Trans Neural Syst Rehabil Eng 20:410-421, 2012

36. Stanslaski S, Cong P, Carlson D, Santa W, Jensen R, Molnar $\mathrm{G}$, et al: An implantable bi-directional brain-machine interface system for chronic neuroprosthesis research. Conf Proc IEEE Eng Med Biol Soc 2009:5494-5497, 2009

37. Starr PA, Christine CW, Theodosopoulos PV, Lindsey N, Byrd D, Mosley A, et al: Implantation of deep brain stimulators into the subthalamic nucleus: technical approach and magnetic resonance imaging-verified lead locations. J Neurosurg 97:370-387, 2002

38. Sun FT, Morrell MJ: The RNS System: responsive cortical stimulation for the treatment of refractory partial epilepsy. Expert Rev Med Devices 11:563-572, 2014

39. Swann NC, de Hemptinne C, Miocinovic S, Qasim S, Wang SS, Ziman N, et al: Gamma oscillations in the hyperkinetic state detected with chronic human brain recordings in Parkinson's disease. J Neurosci 36:6445-6458, 2016

40. Trager MH, Koop MM, Velisar A, Blumenfeld Z, Nikolau JS, Quinn EJ, et al: Subthalamic beta oscillations are attenuated after withdrawal of chronic high frequency neurostimulation in Parkinson's disease. Neurobiol Dis 96:22-30, 2016
41. Truccolo W, Friehs GM, Donoghue JP, Hochberg LR: Primary motor cortex tuning to intended movement kinematics in humans with tetraplegia. J Neurosci 28:1163-1178, 2008

42. Van Gompel JJ, Klassen BT, Worrell GA, Lee KH, Shin C, Zhao CZ, et al: Anterior nuclear deep brain stimulation guided by concordant hippocampal recording. Neurosurg Focus 38(6):E9, 2015

43. Wang DD, de Hemptinne C, Miocinovic S, Qasim SE, Miller AM, Ostrem JL, et al: Subthalamic local field potentials in Parkinson's disease and isolated dystonia: An evaluation of potential biomarkers. Neurobiol Dis 89:213-222, 2016

44. Wichmann T, DeLong MR: Basal ganglia discharge abnormalities in Parkinson's disease. J Neural Transm Suppl (70):21-25, 2006

45. Yousry TA, Schmid UD, Alkadhi H, Schmidt D, Peraud A, Buettner A, et al: Localization of the motor hand area to a knob on the precentral gyrus. A new landmark. Brain 120:141-157, 1997

\section{Disclosures}

Dr. Ostrem has received support from Medtronic and Boston Scientific for non-study-related clinical or research efforts. Dr. Starr has received clinical or research support from Medtronic for the study described, has received support from Boston Scientific for non-study-related clinical or research effort, and holds a patent with the US Patent Office. Implanted devices and technical support were provided at no charge by Medtronic Inc. under a research agreement. Medtronic engineers also reviewed the manuscript for technical accuracy. University of California, San Francisco, holds one patent and has filed another preliminary patent application based on results using Activa PC+S, and Drs. Swann, de Hemptinne, Ostrem, and Starr are co-inventors on both these patents.

\section{Author Contributions}

Conception and design: Swann, de Hemptinne, Ostrem, Starr. Acquisition of data: Swann, de Hemptinne, Miocinovic, Qasim, Galifianakis, San Luciano. Analysis and interpretation of data: Swann, de Hemptinne, Miocinovic. Drafting the article: Swann, Starr. Critically revising the article: all authors. Reviewed submitted version of manuscript: all authors. Approved the final version of the manuscript on behalf of all authors: Swann. Statistical analysis: Swann. Administrative/technical/material support: Wang, Ziman, Taylor. Study supervision: Starr.

\section{Correspondence}

Nicole C. Swann, Department of Neurological Surgery, University of California, San Francisco, 513 Parnassus HSE 8, Rm. 823, San Francisco, CA 94143. email: nicole.swann@ucsf.edu. 